\title{
Comparison of detection of Borrelia burgdorferi DNA and anti-Borrelia burgdorferi antibodies in patients with erythema migrans in north-eastern Poland
}

\author{
Anna Moniuszko ${ }^{1}$, Justyna Dunaj ${ }^{1}$, Joanna Zajkowska ${ }^{1}$, Piotr Czupryna ${ }^{1}$, Renata Świerzbińska ${ }^{1}$, Katarzyna Guziejko ${ }^{1}$, \\ Piotr Aleksiejczuk², Gerald Barry³, Maciej Kondrusik ${ }^{1}$, Sławomir Pancewicz ${ }^{1}$
}

\author{
${ }^{1}$ Department of Infectious Diseases and Neuroinfections, Medical University of Bialystok, Bialystok, Poland \\ Head of Department: Prof. Slawomir Pancewicz MD, PhD \\ ${ }^{2}$ Department of Dermatology and Venereology, Medical University of Bialystok, Bialystok, Poland \\ Head of Department: Prof. Iwona Flisiak MD, PhD \\ ${ }^{3}$ The Roslin Institute and Royal (Dick) School of Veterinary Studies, University of Edinburgh, Easter Bush, Roslin, Scotland, UK \\ Head of Department: Prof. John Fazakerley
}

Postep Derm Alergol 2015; XXXII, 1: 11-14 DOI: 10.5114/pdia.2014.40940

\begin{abstract}
Introduction: Diagnostic methods in erythema migrans are still not standardized.

Aim: To evaluate the frequency of Borrelia burgdorferi s.l. DNA presence in patients with erythema migrans (EM); to assess the polymerase chain reaction (PCR) procedure for detecting B. burgdorferi s.l. DNA in patients with the skin form of Lyme borreliosis; and to compare the results of the PCR-based method with the traditional ELISA method. Material and methods: Skin biopsy and blood samples from 93 patients with EM were examined for B. burgdorferi s.l. DNA detection (PCR). Seventy-one of these patients were examined for the presence of anti-B. burgdorferi s.l. antibodies (ELISA).

Results: Borrelia burgdorferi s.l. DNA was detected in $48 \%$ of the skin biopsy specimens and in $2 \%$ of blood samples. Only 1 patient was PCR positive in both blood and skin samples. Seventy percent of patients whose PCR results were positive were bitten by a tick less than 14 days before. IgM anti-B. burgdorferi s.l - specific antibodies were present in the serum of $35 \%$ of patients and IgG antibodies - in $30 \%$ of patients. Seventeen percent were positive in both IgM and IgG.

Conclusions: Polymerase chain reaction of skin biopsy specimens seems to be currently the most sensitive and specific test for the diagnosis of patients with EM, especially in patients with a short duration of the disease $(<14$ days $)$ but still its effectiveness is much lower than expected. Polymerase chain reaction of blood samples cannot be recommended at the present time for the routine diagnostic of patients with EM.
\end{abstract}

Key words: erythema migrans, Borrelia burgdorferi, polymerase chain reaction, ELISA.

\section{Introduction}

Erythema migrans (EM) is a skin lesion which usually appears near a tick bite as a feature of early Lyme disease (LD). In adults EM is often localized on the limbs and trunk and in children in the head and neck region. According to the American Centers for Disease Control and Prevention (CDC), EM is defined as a skin lesion that typically begins as a red macule or papule to form a large round lesion, often with partial central clearing. A solitary lesion diameter must reach at least $5 \mathrm{~cm}$ in size [1]. However in the literature the so-called miniEM is also described (diameter less than $5 \mathrm{~cm}$ ) [2]. Secondary lesions may also occur. Annular erythematous lesions occurring within several hours after a tick bite represent hypersensitivity reactions and do not qualify as EM. For most patients, the expanding EM lesion is accompanied by intermittent acute symptoms, particularly fatigue, fever, headache, mild neck stiffness, arthralgia or myalgia.

Early implementation of antibiotic therapy may inhibit anti-Borrelia burgdorferi s.l. antibody production. If not

Address for correspondence: Anna Moniuszko MD, PhD, Department of Infectious Diseases and Neuroinfections, Medical University of Bialystok, 14 Zurawia St, 15-540 Bialystok, Poland, phone: +48 8574095 14, fax: +48 8574095 15, e-mail: annamoniuszko@op.pl Received: 2.09.2013, accepted: 24.10.2013. 
treated, EM disappears spontaneously in approximately 4 weeks post infection [3]. However treatment is required to prevent development of later stages of the disease.

Laboratory confirmation is recommended for persons with no known exposure to a tick bite. The diagnostic process of LD diagnosis setting consists of two steps: basic serological/immunoenzymatic ELISA followed by confirmation with Western blot or Immunoblot. These methods are not useful in EM diagnosis because of a delay in anti-B. burgdorferi s.l. antibody production (6-8 weeks after infection) $[4,5]$. Therefore, the effectiveness of this methodology for use in diagnostics is limited and other diagnostic techniques are required. Although, polymerase chain reaction (PCR) seems to be especially useful in EM, because of the coincidence of skin lesions and bacteremia - spirochetemia [6], which may be the cause of general symptoms, up to date, PCR-based methods are not widely used in LD diagnosis as it would be expected.

\section{Aim}

The aim of the study was to evaluate the frequency of $B$. burgdorferi s.l. DNA presence in patients with EM, to assess the effectiveness of PCR at detecting Borrelia DNA in the skin form of LD and to compare the results of PCRbased methodology with the traditional ELISA methods.

\section{Material and methods}

The study included 93 patients admitted to the Department of Infectious Diseases and Neuroinfections of the Medical University of Bialystok, Poland, diagnosed clinically with EM. The study group was composed of 37 women and 56 men with a mean age of $48 \pm 15$ years.

Sixty-eight (73\%) patients remembered a tick bite. The mean time of the tick bite to onset of symptoms was $2.2 \pm 2.4$ weeks. The mean diameter of the skin lesion was $14.3 \pm 8 \mathrm{~cm}$. Four (5\%) patients had multiple EM at the same time. Twenty-nine (31\%) patients suffered from headache, 25 (26\%) from muscle pain, 13 (14\%) from joints pain, 27 (28\%) from tiredness and 14 (15\%) had fever. Polymerase chain reaction examinations were carried once, at the moment of diagnosis, before treatment. All patients signed agreement to take part in research.

Punch skin biopsy samples of $3 \mathrm{~mm}$ in diameter from the expanding edge of the lesion and whole blood samples were taken from all patients and examined for the presence of B. burgdorferi s.l. DNA by nPCR (nested $P(R)$. Additionally 71 of the same patients were examined 6 weeks later for the presence of anti-B. burgdorferi s.l. IgM and IgG antibodies by ELISA (Recombinant-Borrelia IgM and IgG tests (Biomedica)).

Borrelia burgdorferi s.l. DNA detection was performed by $n P C R$. Bacterial DNA from human skin biopsy and blood samples was isolated in a clean area that had been previously decontaminated by UV light.
After collection, skin biopsy samples were placed in aseptic rounded-bottom tubes and frozen or prepared on fresh. All skin biopsies were cut into smaller parts with stainless steel knife-edge and covered with a maximum of $80 \mu \mathrm{l}$ PBS buffer. Whole blood samples were collected on EDTA and extracted within $24 \mathrm{~h}$.

\section{DNA extraction}

DNA from whole blood and skin biopsies was extracted by using a Qiagen DNAeasy Blood and Tissue Mini kit according to the manufacturer's instructions. Purified DNA $(100 \mu \mathrm{l})$ was then frozen at $-20^{\circ} \mathrm{C}$ before amplification.

\section{Polymearse chain reaction protocol for Borrelia burgdorferi s.l.}

A fragment of the fla gene, the specific DNA sequence encoding flagellin was used for B. burgdorferi s.l. detection in "one tube" nested PCR. The B. burgdorferi PCR kit (GeneProof, Czech Republic) for in vitro diagnostics, which was used for this purpose, minimizing non-specific reactions and maximizing sensitivity because of employing "hot start" technology. Possibility of PCR inhibition is controlled by addition of internal standard into the reaction mix. The risk of contamination is prevented by using uracil-DNA-glycosylase (UDG). Four $\mu$ l of the template DNA isolates was added to $36 \mu$ l of the MasterMix for the final reaction mix volume of $40 \mu \mathrm{l}$. The course of the reaction was performed in accordance with the manufacturer's instructions on the SensoQuest LabCycler (SensoQuest, Germany) with authors' own modifications. Nested PCR was performed in the following amplification program: UDG decontamination, initial denaturation at $96^{\circ} \mathrm{C}$ for $10 \mathrm{~min}$, first amplification for 30 cycles (denaturation at $96^{\circ} \mathrm{C}$ for $20 \mathrm{~s}$, annealing at $68^{\circ} \mathrm{C}$ for $20 \mathrm{~s}$, extension at $72^{\circ} \mathrm{C}$ for $40 \mathrm{~s}$ ), second amplification for 45 cycles (denaturation at $96^{\circ} \mathrm{C}$ for $20 \mathrm{~s}$, annealing at $54^{\circ} \mathrm{C}$ for $20 \mathrm{~s}$, extension at $72^{\circ} \mathrm{C}$ for $30 \mathrm{~s}$ ) and final extension at $72^{\circ} \mathrm{C}$ for $2 \mathrm{~min}$. The samples were cooled at $+4^{\circ} \mathrm{C}$.

The PCR products were separated on $2 \%$ agarose gel (Sigma-Aldrich, Germany) with the addition of ethidium bromide ( $5 \mu \mathrm{g} / \mathrm{ml}$; Syngen, USA) at $80 \mathrm{~V}$ for $80 \mathrm{~min}$. The results of the PCR were viewed under UV light (UV to Gel Logic System 100 (Kodak Imaging System, Inc., USA)). Probes with the PCR product in size of 276 base pairs (bp) were regarded as positive. The internal control had a size of $420 \mathrm{bp}$. For precise detection of $B$. burgdorferi s.l. fla amplicons and internal control molecular weight marker (M100-500-Blirt S.A. Poland) was used.

\section{Statistical analysis}

Statistical analysis was performed using StatSoft Statistica 10.0. Patients were divided into 2 groups, depending on PCR test results (group I- positive PCR in the skin sample, group II - negative PCR in the skin sample). Groups were compared using U-Mann-Whitney test. Values of $p<0.05$ was considered as statistically significant. 


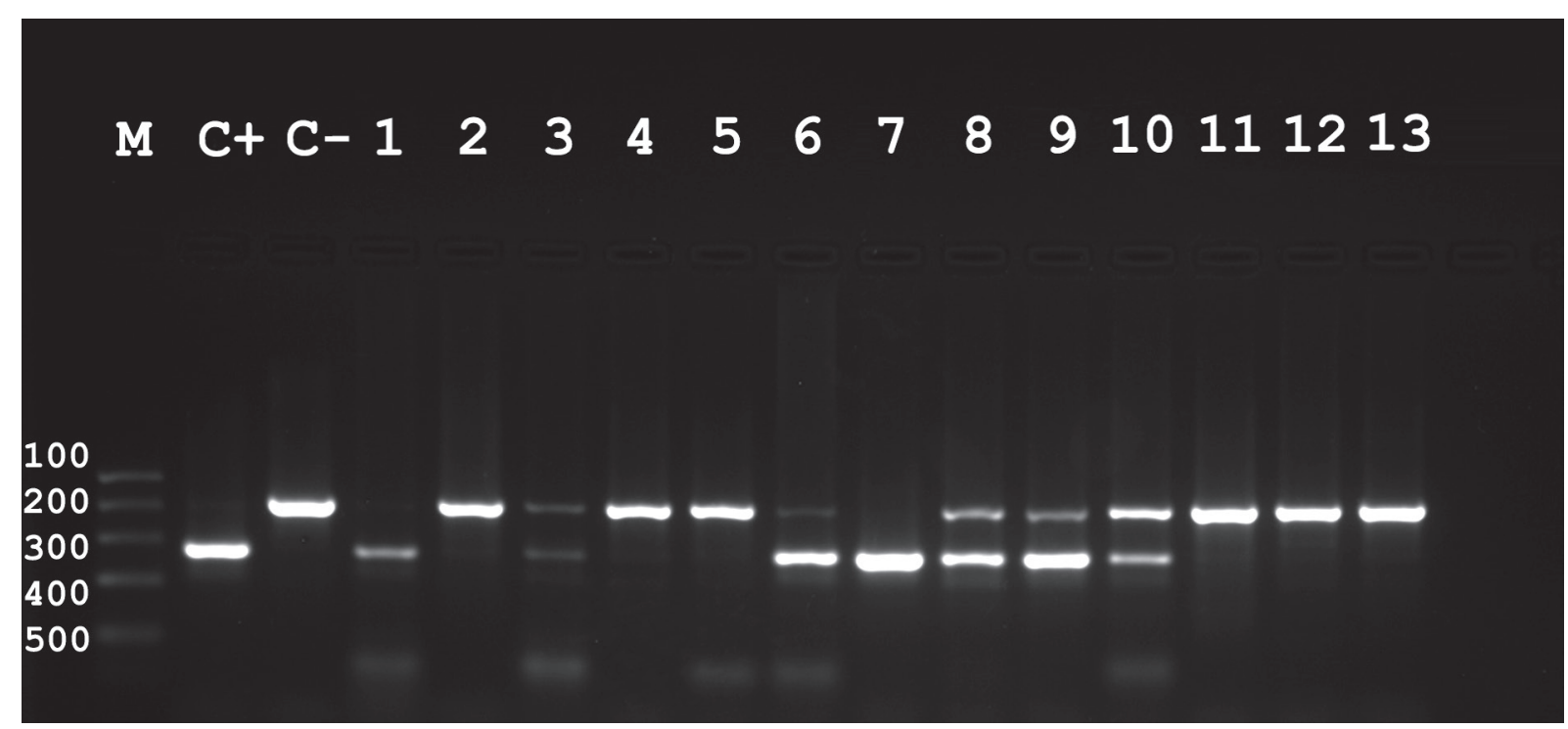

Figure 1. Electrophoresis results of Borrelia burgdorferi s.l. detection in the skin sample

$C_{+}-$positive control, $C--$ negative control, $M$ - molecular weight marker, fla gene - $276 \mathrm{bp}$, internal control - $420 \mathrm{bp}$, molecular weight marker-100-500 bp, positive samples: 1, 3, 6, 7, 8, 9, 10; negative samples: 2, 4, 5, 11, 12, 13.

\section{Results}

Specific B. burgdorferi DNA was detected in $48 \%$ of the skin biopsy specimens and in $2 \%$ of the blood samples from patients with EM (example in Figure 1). Only in 1 patient (1\%) the results were positive either in a skin or blood sample. Six weeks after PCR examination IgM anti-B. burgdorferi-specific antibodies were present in serum of $35 \%$ of patients and IgG antibodies - in 30\% of patients. Sixteen (17\%) patients were positive in both classes. In $70 \%$ of PCR positive patients, duration of the disease was shorter than $<14$ days.

We compared a group of patients with positive PCR in the skin sample with patients with negative PCR in the skin sample, regarding ELISA tests results, diameter of EM, period from the tick bite. No statistically significant differences were found. We also compared patients with positive ELISA tests results and negative ELISA tests results regarding the diameter of EM and the period from the tick bite. No statistically significant differences were found.

\section{Discussion}

The usefulness of PCR in the diagnosis of LD is widely discussed. Effectiveness of the PCR method in detection of DNA of B. burgdorferi: B. burgdorferi sensu stricto, $B$. garinii, $B$. afzelii in various human fluids (blood, csf, urine) has been shown by many previous studies [7-9], but in the case of LD, the clinical efficacy of this method has not been equivocally approved [10]. The probability of false positive results is high. According to Bukinis and Barbour, PCR will also detect the presence of DNA from dead spirochetes. While increasing detection sen- sitivity, this also decreases the usefulness of this method in the diagnosis of an active infection [11]. Michel et al. have already described a method of identification and differentiation of 5 genospecies of B. burgdorferi s.l. (B. burgdorferi sensu stricto, B. afzelii, B. garinii, B. valaisiana, and $B$. lusitaniae), based on PCR of the surface antigen - OspA [12].

Goodman et al. showed that the sensitivity of PCR in spirochetemia detection is $18.4 \%$, but if the patient displays any general symptoms of LD, the detection rate is higher (30.3\%) and in the case of multiple skin lesions, it is as high as 37.5\% [13].

Schwartz et al. observed that the sensitivity of PCR in skin lesions is 59\% [14]. Nowakowski et al. compared sensitivity of various diagnostic methods in patients with EM and concluded that the most sensitive method is amplification of DNA from skin change (80.2\%). The culture of spirochetes from skin lesions and blood presented lower sensitivity (51.1\% and $44.7 \%$, respectively) [15]. Wormser et al. detected in $50 \%$, cultures of spirochetes from peripheral blood of patients with EM [16]. Liveris et al. evaluated the sensitivity of 5 direct diagnostic methods (culture and nested PCR of a 2-mm skin biopsy specimen, nested PCR and quantitative PCR (qPCR)) and observed that results of one or more of these tests were positive in $93.9 \%$ of the patients. The results of this study demonstrate that direct detection methods such as PCR and culture are highly sensitive in untreated adult patients with EM [17]. These observations are not in accordance with results of our study.

Tylewska-Wierzbanowska and Chmielewski compared the diagnostic effectiveness of serological methods and 
PCR and came to the conclusion that PCR positive samples of blood, cerebrospinal fluid and joint fluid usually showed a low concentration or lack of antibodies against B. burgdorferi [18]. Also in our study the percentage of IgM and IgG positive ELISA tests was lower than positive results for PCR from skin specimens.

The reasons influencing the limitations of PCR in the diagnosis of EM include huge variability of $B$. burgdorferi s.l., what causes difficulties in specification of genes and short time of presence of bacteria in body fluids, e.g. in blood. Because of these reasons, negative PCR results do not exclude an infection with $B$. burgdorferi. However, our results show that PCR based diagnosis would be useful as a diagnostic tool during the early phase of the disease, especially when there is no possibility of antibody detection. It has been suggested that to achieve a sufficient diagnostic sensitivity of PCR it is recommended to use minimally two amplification systems in parallel [19].

Importantly however, PCR positivity cannot be used as a measure of LD treatment effectiveness due to the long persistence of bacterial DNA after antibiotic treatment.

\section{Conclusions}

Based on the results of our study we conclude that PCR of skin biopsy specimens in patients with a short duration of the disease ( $<14$ days) currently seems to be the most sensitive and specific test for the diagnosis of patients with EM, but still its effectiveness is much lower than expected. Polymerase chain reaction of blood samples cannot be recommended at the present time for the routine diagnostic of patients with EM.

\section{Conflict of interest}

The authors declare no conflict of interest.

\section{References}

1. Stanek G, Fingerle V, Hunfeld KP, et al. Lyme borreliosis: clinical case definitions for diagnosis and management in Europe. Clin Microbiol Infect 2011; 17: 69-79.

2. Weber K, Wilske B. Mini erythema migrans - a sign of early Lyme borreliosis. Dermatology 2006; 212: 113-6.

3. Pancewicz SA, Januszkiewicz A, Hermanowska-Szpakowicz T. Clinical picture of Lyme disease in own material. Klinika 1997; 8: 420-3.

4. Weber K. Aspects of Lyme borreliosis in Europe. Eur J Clin Microbiol Infect Dis 2001; 20: 6-13.

5. Ziuzia IR, Efimova NS, Vorobcaeva NN, et al. Clinical and morphological characteristics of migrating erythrema in patients with Ixodes tick-borne Lyme disease. Med Parazitol (Mosk) 1999; 4: 36-41.

6. Maraspin V, Ogrinc K, Ruzic-Sabljić E, et al. Isolation of Borrelia burgdorferi sensu lato from blood of adult patients with borrelial lymphocytoma, Lyme neuroborreliosis, Lyme arthritis and acrodermatitis chronica atropicans. Infection 2011; 39: 35-40.
7. Oksi J, Marttila H, Soini H, et al. Early dissemination of Borrelia burgdorferi without generalized symptoms in patients with erythema migrans. APMIS 2001; 109: 581-8.

8. Zore A, Ruzic-Sabljis E, Maraspin V, et al. Sensitivity of culture and polymerase chain reaction for the etiologic diagnosis of erythema migrans. Wien Klin Wochenschr 2002; 114: 606-9.

9. Pícha D, Moravcová L, Holecková D, et al. Examination of specific DNA by PCR in patients with different forms of Lyme borreliosis. Int J Dermatol 2008; 47: 1004-10.

10. Lange R, Seyyedi S. Evidence of a Lyme borreliosis infection from the viewpoint of laboratory medicine. Int J Med Microbiol 2002; 291: 120-4.

11. Bunikis J, Barbour AG. Laboratory testing for suspected Lyme disease. Med Clin N Am 2002; 86: 311-39.

12. Michel H, Wilske B. An OspA-polymerase chain reaction/restriction fragment length polymorphism-based method for sensitive detection and reliable differentiation of all European Borrelia burgdorferi sensu lato species and OspA types. Med Microbiol Immunol 2004; 193: 219-26.

13. Goodman JL, Bradley JF, Ross AE, et al. Bloodstream invasion in early Lyme disease: results from a prospective, controlled, blinded study using the polymerase chain reaction. Am J Med 1995; 99: 6-12.

14. Schwartz I, Wormser GP, Schwartz JJ, et al. Diagnosis of early Lyme disease by polymerase chain reaction amplification and culture of skin biopsies from erythema migrans lesions. J Clin Microbiol 1992; 30: 3082-8.

15. Nowakowski J, Schwartz I, Liveris D, et al. Laboratory techniques for patients with early Lyme disease associated with erythema migrans: a comparison of different techniques. Clin Infec Dis 2001; 15: 2023-7.

16. Wormser GP, Bittker S, Cooper D, et al. Comparison of yield of blood cultures using serum or plasma from patients with early Lyme disease. J Clin Microbiol 2000; 38: 1648-50.

17. Liveris D, Schwartz I, McKenna D, et al. Comparison of five diagnostic modalities for direct detection of Borrelia burgdorferi in patients with early Lyme disease. Diagn Microbiol Infect Dis 2012; 73: 243-5.

18. Tylewska-Wierzbanowska S, Chmielewski T. Limitation of serological testing for Lyme borreliosis: evaluation of ELISA and western blot in comparison with PCR and culture methods. Wien Klin Wochenschr 2002; 114: 601-5.

19. Moravcová L, Pícha D, Vanousová D, et al. Detection of borrelia DNA from patients with neuroborreliosis and erythema migrans. Klin Mikrobiol Infekc Lek 2009; 15: 160-5. 